Rev Biomed 2005; 16:227-237.

\title{
Parasitosis intestinales en escolares: relación entre su prevalencia en heces y en el lecho subungueal.
}

\section{Artículo Original}

Fadia Al Rumhein, Julia Sánchez, Ixora Requena, Ytalia Blanco, Rodolfo Devera.

Grupo de Parasitosis Intestinal, Dpto. de Parasitología y Microbiología, Escuela de Ciencias de la Salud, Universidad de Oriente, Ciudad Bolívar, Estado Bolívar, Venezuela.

\section{RESUMEN.}

Introducción. Los parásitos intestinales de forma general son transmitidos a través de la ingestión de agua y/o alimentos contaminados con formas infectantes. También se ha llamado la atención acerca del papel del lecho subungueal como posible diseminador de enteropatógenos en personas con hábitos de higiene inadecuados.

Objetivo. Determinar la prevalencia de parásitos intestinales en las heces y en el lecho subungueal en escolares de la U.E.E. Teresa de la Parra del Barrio Buen Retiro en San Félix, Estado Bolívar, Venezuela.

Material y Métodos. De mayo a julio de 2003 fueron evaluados 344 niños, entre 6 y 15 años. De cada uno fue obtenida una muestra de heces y analizada mediante la técnica de examen directo y los métodos de concentración de Willis y formoléter. También fueron colectadas muestras de uñas y material subungueal siendo analizadas mediante la técnica de formol-éter.

Resultados. La prevalencia de parásitos en heces fue de $97.4 \%$ (335/344). No hubo predilección en cuanto a la edad $\left(\chi^{2}=6.2 ;\right.$ g.1. $\left.=4\right)$ o sexo de los parasitados $(\mathrm{p}>0,05)$. Los protozoarios fueron más prevalentes, destacando Blastocystis hominis con $76.2 \%$. Entre los helmintos el más común fue Trichuris trichiura con $74.1 \%$. La prevalencia de estadios parasitarios en el deposito subungueal fue de $3.6 \%$ (11/307), siendo A. lumbricoides y $E$. coli los más comunes. No hubo relación entre la presencia de parásitos en el lecho subungueal y en las heces.

Conclusiones. Se determinó una elevada prevalencia de parasitosis intestinales en los escolares evaluados. Aunque se demostró la importancia del lecho subungueal como diseminador de enteroparásitos, este mecanismo no parece ser el principal implicado en la cadena epidemiológica de estas parasitosis en los niños estudiados. (Rev Biomed 2005; 16:227-237)

Palabras clave: parásitos intestinales, epidemiología, lecho subungueal, Venezuela.

Solicitud de sobretiros: Dr. Rodolfo Devera. Departamento de Parasitología y Microbiología, Escuela de Ciencias de la Salud, Universidad de Oriente, Núcleo Bolívar. Av. José Méndez, Barrio Ajuro, Ciudad Bolívar, Estado Bolívar, Código postal: 8001-A. Venezuela. E-mail: rodolfodevera@hotmail.com

Recibido el 2/Septiembre/2005. Aceptado para publicación el 7/Noviembre/2005.

Este artículo está disponible en http://www.uady.mx/sitios/biomedic/revbiomed/pdf/rb051642.pdf

Vol. 16/No. 4/Octubre-Diciembre, 2005 
F Al Rumhein, J Sánchez, I Requena, Y Blanco, R Devera.

\section{SUMMARY.}

Intestinal parasitosis in schoolchildren: relationship between its prevalence in stool and in the subungueal area.

Introduction. Intestinal parasites are frequently transmitted by water and/or food ingestion contaminated with infective organisms. The role of subungueal deposits as disseminators of enteropatogens in people with deficient hygienic habits has been discussed.

Objective. To determine the prevalence of intestinal parasites in stool and in subungueal deposits in schoolchildren from the U.E.E. Teresa de la Parra, Buen Retiro neighborhood, San Félix, Bolívar State in Venezuela.

Material and methods. A cross-sectional study was carried out from May to July 2003 on 195 children of both sexes, aged 6-15 years. A stool sample was obtained from each child and analyzed by the direct examination technique and by the Willis and formol-ether concentration methods. Samples of nails and deposit under nails were also collected and processed by the formol-ether method.

Results. The prevalence of parasites in stool was $97.4 \%(335 / 344)$. The parasitosis did not show age $\left(\chi^{2}=6.2\right.$; f.d. $\left.=4\right)$ or sex $(\mathrm{p}>0.05)$ preferences. Protozoans were more frequent than helminthes. The more common protozoan was Blastocystis hominis with $76.2 \%$. The most common helminth was Trichuris trichiura with $74.1 \%$. The parasitic stages prevalence in subungueal deposits was $3.6 \%$ (11/307), A. lumbricoides and E. coli being the most frequent. There was no relationship between the parasite presence in stool and in the subungueal deposits.

Conclusion. A high prevalence of intestinal parasitosis in the school children evaluated was observed. Although the importance of subungueal deposits as disseminators of enteroparasites was demonstrated, this mechanism does not appear to be the major factor implicated in the epidemiological chain of parasitosis in these children.

(Rev Biomed 2005; 16:227-237)
Key words: intestinal parasitosis, epidemiology, subungueal area, Venezuela.

\section{INTRODUCCIÓN.}

Las parasitosis intestinales siguen constituyendo un problema de salud pública para los habitantes de diversas regiones del mundo y en especial en áreas tropicales y subtropicales. La población principalmente afectada sigue siendo la infantil debido a su inmadurez inmunológica y poco desarrollo de hábitos higiénicos. Los parásitos intestinales pueden llevar a consecuencias negativas, tanto físicas como desde el punto de vista cognitivo, en muchos niños parasitados (1-7).

La mayoría de los parásitos intestinales son transmitidos por vía fecal-oral, especialmente ingestión de agua y/o alimentos contaminados con formas infectantes. Esta contaminación puede ocurrir directamente por deficientes prácticas higiénicas de manipuladores de alimentos infectados o indirectamente a través de la ingestión de agua contaminada u otras vías de contaminación cruzadas (8-10). Los geohelmintos requieren de un proceso de maduración en el suelo para poder infectar a otro hospedero y pueden hacerlo activamente a través de larvas que penetran la piel $(1,8,11)$. Otros mecanismos de infección, llamados alternativos, también han sido sugeridos y en los cuales intervendrían factores como higiene personal inadecuada y elevada carga de formas infectantes. Uno de estos mecanismos es el empleo de fomites o utensilios $(12,13)$, debido a la conocida resistencia de los huevos de helmintos y quistes de protozoarios a las condiciones ambientales (8). También se ha llamado la atención acerca del papel del lecho subungueal como posible diseminador de enteropatógenos en personas con hábitos de higiene inadecuados $(14,15)$.

El primer enteroparásito que fue buscado sistemáticamente en el lecho subungueal humano fue Enterobius vermicularis $(14,16)$, de hecho fue la principal técnica diagnostica hasta que otras metodologías fueron introducidas (17). Después fueron observados quistes de Entamoeba

\section{Revista Biomédica}




\section{Parasitosis intestinales en escolares.}

histolytica por Andrews (18). Sin embargo, era obvio pensar que huevos de otros helmintos y quistes de protozoarios pudiesen ser observados en el material que se acumula debajo del borde libre de las uñas de aquellos individuos que no cumpliesen con las debidas nociones de higiene y particularmente los niños por su inmadurez de hábitos higiénicos. Lo que se necesitaba era de una técnica eficaz para su demostración $(14,15)$. Goulart y col. (14), modificaron la técnica de Ritchie adaptándola al estudio de ese material subungueal, demostrando además de la presencia de los parásitos anteriormente citados, quistes de Giardia lamblia y huevos de Taenia, Trichuris trichiura y Ascaris lumbricoides. Luego, otros autores, aplicando ésta y otras técnicas similares han demostrado la presencia de huevos de ancilostomideos (15, 19), Hymenolepis nana (Rincón de Heredia, 1983), quistes de Entamoeba coli (15) y Endolimax nana (20) en el depósito subungueal.

Otros autores han encontrado estas diferentes especies de helmintos y protozoarios con cifras de prevalencia generalmente elevadas, en diferentes grupos de poblaciones, comprobando la importancia del lecho subungueal como diseminador de enteroparásitos (12, 15, 19-23).

Con la finalidad de contribuir al conocimiento de la epidemiología de las parasitosis intestinales en nuestro medio, se realizó un estudio para determinar la prevalencia de parasitosis intestinales en un grupo de escolares, estableciendo relaciones entre las prevalencias de parásitos en las heces y en el depósito subungueal de los niños y de esta forma valorar la importancia del lecho subungueal como fuente de infección.

\section{MATERIAL Y MÉTODOS.}

1. Área de estudio. Se realizó un estudio de tipo seccional entre mayo y julio de 2003 en escolares matriculados en la Unidad Educativa Estadal (U.E.E.) Teresa de la Parra, ubicada en el barrio Buen Retiro, Municipio Caroní, al norte del estado Bolívar, en la periferia de la ciudad de San
Félix. El Barrio Buen Retiro se divide en tres sectores. El sector 3 es producto de un proceso de invasión irregular, no cuenta con servicio de aseo urbano, cloacas ni calles asfaltadas, la energía eléctrica es deficiente así como el suministro de agua potable. El sector 1 es el más extenso y con mayor número de viviendas, mientras que el sector 2 es más reducido territorialmente y también el más antiguo, siendo el que presenta las mejores condiciones de saneamiento ambiental.

De una manera global se trata de una comunidad de tipo suburbano, con bajas condiciones socio-económicas. Aunque la mayoría de sus calles están pavimentadas (sectores 1 y 2), muchas están deterioradas; existen todos los servicios públicos con excepción de la red de cloacas, por lo que muchas familias utilizan pozos sépticos. El saneamiento ambiental es deficiente aunque no deplorable. Aunque hay red de agua por tuberías el servicio es irregular. La recolección de basura se realiza dos veces por semana de forma irregular y apenas en las calles principales, por ello en muchas viviendas la basura es colocada al aire libre o es quemada.

2. Universo. El universo estuvo representado por el total de alumnos matriculados para el periodo 2002-2003, que, incluyendo del 1ero al 6o grado, eran 1189 niños distribuidos en 32 secciones, 16 en la mañana y 16 en la tarde.

3. Muestra. Estuvo formada por aproximadamente el 30\% del universo. Esta muestra fue seleccionada escogiendo aleatoriamente de cada sección 20 niños. Para ello de cada 2 alumnos de la lista total de cada sección se seleccionó uno, hasta completar 20 por sección. Esos niños fueron citados junto con su representante y se les entregó un envase recolector de heces e instrucciones para obtener la muestra.

\section{Recolección de datos.}

4.1. Datos de Identificación. Se obtuvo en consentimiento escrito de cada padre o representante para participar del estudio. De cada niño seleccionado se investigaron datos de identificación, edad y sexo, dirección, etc., mediante 


\section{F Al Rumhein, J Sánchez, I Requena, Y Blanco, R Devera.}

una ficha de control diseñada para tal fin.

\subsection{Recolección de Muestras.}

a. Heces. Una porción de la muestra fecal obtenida por evacuación espontánea fue analizada en la propia escuela mediante el método de examen directo con solución salina $0,85 \%$ y lugol, y el método de concentración de Willis (24). Otra porción de la muestra fue preservada en formol al $10 \%$ en envase adecuado, rotulada y trasladada al laboratorio y posteriormente se analizó mediante la técnica de formol-éter (24). Para su ejecución, el agua destilada fue sustituida por solución salina fisiológica $0,85 \%$ para evitar la destrucción de los trofozoitos de Blastocystis hominis.

Por razones éticas y sociales, le fueron realizados los exámenes a todo niño que aun no siendo seleccionado, sus padres o representantes así lo solicitaron. Sin embargo, ellos no fueron incluidos en el estudio.

\section{b. Material del lecho subungueal.}

La técnica empleada para la colecta del contenido subungueal fue una adaptación de las empleadas por Goulart y col. (14) y Campos (15). Para ello se raspó cuidadosamente con un palillo de madera el material localizado debajo de las uñas de las manos de cada escolar, posteriormente se cortaron las uñas y ambos materiales se colocaron en frascos viales con tapa de rosca conteniendo $10 \mathrm{ml}$ de formol al 10\%, los cuales estaban previamente identificados.

Después de cortar las uñas y antes de ser usados en otro niño, los materiales (corta uñas y tijeras) fueron lavados con ayuda de un cepillo de cerdas de nylon en alcohol isopropílico al 95\%, después pasadas por agua destilada y finalmente secadas con una gasa limpia.

Antes de hacer el análisis del material colectado se agitó vigorosamente el contenido de los frascos con ayuda de una varilla de vidrio, para garantizar la disgregación de todo el material. Se pasó ese líquido por gasa doblada en cuatro y luego fue colocado en un tubo de centrifuga de $15 \mathrm{ml}$ (14). Se adicionó $1 \mathrm{ml}$ de éter y se procedió como en el método de formol-éter (24), es decir, se agitó vigorosamente por 30 segundos, centrifugación a 2.500 r.p.m. por 2 minutos. Después se realizó la decantación de las tres capas superiores formadas en el tubo. El sedimento (última capa inferior) fue examinada al microscopio empleando lugol, para obtener mejores resultados se examinó todo el sedimento. Todas las preparaciones fueron examinadas al microscopio, por dos observadores diferentes, primero con objetivo de 10X y luego con $40 X$.

\section{Análisis de los datos.}

Para el análisis de los resultados se construyó una base de datos y posteriormente fue analizada con el auxilio del programa SPSS versión 8,0 para Windows. Se utilizaron frecuencias relativas (\%) y los resultados se presentan en tablas. Se usó la prueba $\mathrm{Ji}$ al cuadrado $\left(\chi^{2}\right)$ con un margen de seguridad de $95 \%$ para demostrar la independencia entre las variables: parasitosis, sexo y edad; así como para verificar la relación parásitos en heces versus parásitos en el lecho subungueal (25).

\section{RESULTADOS.}

Entre mayo y julio de 2003 se realizó el estudio coproparasitológico en 344 muestras fecales y 307 muestras de depósito subungueal provenientes de niños, de ambos sexos, en edad escolar matriculados en la U.E.E Teresa de la Parra del Barrio Buen Retiro, San Félix, Estado Bolívar. El total de niños matriculados para el periodo escolar 2002-2003 fue de 1189, por lo tanto la muestra estudiada fue de 28,9\% (344/1189). La media de edad de los 344 niños estudiados fue de 9,3 con una desviación estándar de 1,9. Un total de 162 $(47,1 \%)$ eran niños y 182 niñas $(52,9 \%)$, siendo la muestra homogénea en cuanto al sexo.

La prevalencia de parásitos en las muestras fecales fue de 97,4\% (335/344). En el grupo etário de 8 a 9 años se encontró el mayor número de parasitados, sin embargo, esa diferencia no fue estadísticamente significativa $\left(\chi^{2}=6,19, \mathrm{p}>0,05\right)$ (cuadro 1). Con relación al sexo tampoco hubo diferencias ( $p>0,05)$ ya que el $96,7 \%$ de las niñas resultaron parasitadas mientras que de los niños el

\section{Revista Biomédica}


Parasitosis intestinales en escolares.

Cuadro 1

Escolares con y sin parasitosis según edad, U.E.E. Teresa de la Parra, San Félix, Edo. Bolívar, Venezuela.

\begin{tabular}{|c|c|c|c|c|c|c|}
\hline \multirow{3}{*}{$\begin{array}{l}\text { Grupos } \\
\text { etarios }\end{array}$} & \multicolumn{4}{|c|}{ Escolares } & \multirow{2}{*}{\multicolumn{2}{|c|}{ TOTAL }} \\
\hline & \multicolumn{2}{|c|}{ Parasitados } & \multicolumn{2}{|c|}{ No Parasitados } & & \\
\hline & $\mathbf{N}^{\circ}$ & $\%$ & $\mathbf{N}^{\circ}$ & $\%$ & $\mathbf{N}^{\circ}$ & $\%$ \\
\hline $6-7$ & 73 & 21,2 & 1 & 0,3 & 74 & 21,5 \\
\hline 8 - 9 & 106 & 30,8 & 2 & 0,6 & 108 & 31,4 \\
\hline $10-11$ & 99 & 28,8 & 6 & 1,7 & 105 & 30,5 \\
\hline $12-13$ & 56 & 16,3 & 0 & 0 & 56 & 16,3 \\
\hline 14-15 & 1 & 0,3 & 0 & 0 & 1 & 0,3 \\
\hline TOTAL & 335 & 97,4 & 9 & 2,6 & 344 & 100,0 \\
\hline
\end{tabular}

$\chi^{2}=6,19$ g.l $1=4 \mathrm{p}>0,05$

98,1\% estaba parasitado.

Un total de 14 especies de enteroparásitos y/o comensales fueron diagnosticados. De los parasitados el 16,4\% (55/335) estaba monoparasitado, mientras que $83,6 \%$ resultó poliparasitado (280/335). Las asociaciones protozoarios-helmintos fueron más frecuentes $(70,1 \%)$ que las parasitosis exclusivas por helmintos $(17,6 \%)$ o por protozoarios $(12,2 \%)$. Entre los protozoarios los más prevalentes fueron B. hominis $(76,2 \%)$ у Entamoeba coli $(26,5 \%)$, mientras que entre los helmintos Trichuris trichiura (74,1\%) y Ascaris lumbricoides (48,2\%) resultaron más prevalentes (cuadro 2 ).

En las muestras de depósito subungueal la prevalencia de estadios parasitarias fue de 3,6\%, representado por 11 casos. De ellos el 2,1\% correspondió a protozoarios y $1,5 \%$ a helmintos. Cabe resaltar que en 37 escolares no se tomó muestra de material subungueal por éste ser insuficiente (uñas muy cortas). De los 11 casos positivos, en ocho se identificó una especie parasitaria, mientras que en tres casos habían dos especies. A. lumbricoides fue el parásito más prevalente con cinco casos, seguido de E. coli con cuatro (cuadro 2). Entre los helmintos sólo se observaron huevos y entre los protozoarios

\section{Cuadro 2}

Prevalencia de parásitos intestinales en escolares de la U.E.E. Teresa de la Parra, San Félix, Edo. Bolívar, Venezuela

\begin{tabular}{lcccc}
\hline ESPECIES & \multicolumn{2}{c}{$\begin{array}{c}\text { Heces } \\
(\mathbf{n = 3 4 4})\end{array}$} & $\begin{array}{c}\text { Lecho subungueal } \\
(\mathbf{n = 3 0 7})\end{array}$ \\
\cline { 2 - 5 } & $\mathbf{N}^{\circ}$ & $\mathbf{\%}$ & $\mathbf{N}^{\circ}$ & $\mathbf{\%}$ \\
\hline HELMINTOS & & & & 0,3 \\
Trichuris trichiura & 255 & 74,1 & 1 & 1,6 \\
Ascaris lumbricoides & 166 & 48,3 & 5 & 0 \\
Ancilostomideos & 26 & 7,6 & 0 & 0 \\
Strongyloides stercoralis & 14 & 4,1 & 0 & 0 \\
Enterobius vermicularis & 7 & 2,0 & 0 & 0 \\
Hymenolepis nana & 6 & 1,7 & 0 & 0 \\
PROTOZOARIOS & & & & 1,3 \\
Blastocystis hominis & 262 & 76,2 & 0 & 1,0 \\
Entamoeba coli & 91 & 26,4 & 4 & 0,6 \\
Endolimax nana & 85 & 24,7 & 3 & 0 \\
Giardia lamblia & 79 & 23,0 & 2 & 0 \\
Chilomastix mesnilii & 27 & 7,8 & 0 & 0 \\
Pentatrichomonas hominis & 23 & 6,7 & 0 & 0 \\
Iodamoeba butschlii & 15 & 4,3 & 0 & 0 \\
Entamoeba histolytica/E. dispar & 12 & 3,3 & 0 & 0 \\
\hline
\end{tabular}


F Al Rumhein, J Sánchez, I Requena, Y Blanco, R Devera.

únicamente quistes. De los cinco casos de $A$. lumbricoides, en tres se observó un sólo huevo, en otro dos y en el quinto caso se identificaron cuatro huevos. Muchos de estos huevos conservaban la estructura característica y en un caso se observó la larva en el interior del mismo. Con relación a $E$. coli, de los cuatro casos diagnosticados, en tres se evidenció un solo quiste, y en el cuarto dos, estando ambos bien conservados manteniendo su estructura característica. En el caso de Giardia lamblia, de los dos casos, en uno habían cuatro quistes bien conservados y en el otro apenas un quiste parcialmente deformado (cuadro 3).

Al estudiarse la relación entre los casos de parásitos en las muestras fecales y el material subungueal de los escolares se verificó que los 11 casos con formas parasitarias en el depósito subungueal también tenían parásitos en las heces, sin embargo, esa relación no fue estadísticamente significativa $\left(\chi^{2}=0,30\right.$ g.1. $\left.=1 \mathrm{p}>0,05\right)$ (cuadro 4). Tampoco hubo relación entre la especie parasitaria encontrada en las uñas y la presente en las heces, ya que en 8 casos el parásito que fue identificado en el lecho subungueal no estaba presente en las heces de los niños. De los cuatro casos con $E$. coli ninguno tenía el parásito en las heces y de los cinco casos con A. lumbricoides sólo en dos se demostraron los huevos del helminto en las heces.

\section{DISCUSIÓN.}

Se determinó una elevada prevalencia de parasitosis intestinal $(97,4 \%)$ en los escolares evaluados, lo que coincide con los resultados obtenidos por otros investigadores en escolares de diferentes regiones de Venezuela, principalmente en comunidades rurales (26-30). Ello demuestra las altas tasas de prevalencia de parásitos intestinales en la población infantil venezolana. Con relación al estado Bolívar este resultado es superior al señalado en escolares del área urbana de Ciudad Bolívar (31) y en otras localidades del estado (32). Cabe resaltar que la comunidad de Buen Retiro, donde se ubica la escuela, es de tipo suburbana con bajas condiciones socio-económicas (datos no presentados).

Como en otros estudios, en la presente investigación no se detectó asociación estadísticamente significativa entre las variables parasitosis, sexo y edad de los niños (27-34), aunque la mayor

\section{Cuadro 3}

Escolares con presencia de parásitos en el depósito subungueal según número y aspecto morfológico, U.E.E. Teresa de la Parra, San Félix, Edo. Bolívar, Venezuela.

\begin{tabular}{llll}
\hline \multirow{2}{*}{ Parásito } & $\mathbf{N}^{\circ}$ de & \multicolumn{2}{c}{ Estadios Parasitarios } \\
\cline { 3 - 4 } & Casos & $\mathbf{N}^{\circ}$ y tipo & Aspecto Morfológico \\
\hline \multirow{2}{*}{ Ascaris lumbricoides } & 1 & 2 huevos & Conservado \\
& 2 & 1 huevo & Deforme \\
& 3 & 1 huevo & Larvado \\
Trichuris trichiura & 4 & 4 huevos & Conservado \\
Giardia lamblia & 5 & 1 huevo & Decorticado \\
& 1 & 1 huevo & Conservado \\
Entamoeba coli & 2 & 4 quistes & Conservado \\
& 1 & 1 quiste & Deformado \\
& 2 & 1 quiste & Conservado \\
& 3 & 1 quiste & Deformado \\
Endolimax nana & 4 & 1 quiste & Deformado \\
& 1 & 2 quiste & Deformado \\
& 2 & 1 quiste & Conservado \\
\hline
\end{tabular}




\section{Parasitosis intestinales en escolares.}

Cuadro 4

Relación entre presencia de parásitos en heces y en el material subungueal en escolares de la U.E.E. Teresa de la Parra, San Félix, Edo. Bolívar, Venezuela.

\begin{tabular}{lcccccc}
\hline \multirow{2}{*}{$\begin{array}{l}\text { Parásitos } \\
\text { en material } \\
\text { subungueal }\end{array}$} & \multicolumn{3}{c}{$\begin{array}{c}\text { Parásitos en heces } \\
\text { SI }\end{array}$} & \multicolumn{4}{c}{ NO } & \multicolumn{2}{c}{ TOTAL } \\
\cline { 2 - 7 } Si & $\mathbf{N}^{\circ}$ & $\%$ & $\mathbf{N}^{\circ}$ & $\%$ & $\mathbf{N}^{\circ}$ & $\%$ \\
No & 11 & 3,6 & 0 & 0 & 11 & 3,6 \\
Total & 288 & 93,8 & 8 & 2,6 & 296 & 96,4 \\
\hline
\end{tabular}

$\chi^{2}=0,30$ g.l $=1 \mathrm{p}>0,05$

prevalencia de las parasitosis se observó en el grupo etário de 8-9 años (30,8\%), probablemente esto se deba a la poca variación existente en las edades de los niños estudiados ya que pertenecen a un grupo que comparte actividades similares y, en general, no tienen hábitos higiénicos bien establecidos por lo que presentan la misma posibilidad de infección con las formas infectantes de los parásitos que pueden encontrarse en el medio ambiente $(30,36)$.

Los helmintos, en particular los geohelmintos, tuvieron una elevada prevalencia, lo cual se esperada por tratarse de una comunidad con todas las condiciones ecoepidemiológicas para ello (1, $8,11,37)$. Otros estudios realizados en escolares de comunidades rurales y suburbanas han mostrado resultados similares en diversas regiones de Venezuela $(34,38)$.

En el caso de T. trichiura, que fue uno de los geohelmintos de mayor prevalencia $(74,1 \%)$, los resultados coinciden con los de otros autores que lo señalan como el enteroparásito más común en Venezuela $(11,28,34,38-40)$ y el Estado Bolívar $(31,41)$ principalmente en comunidades rurales.

El segundo helminto en prevalencia $(48,2 \%)$ fue $A$. lumbricoides cuya epidemiología es similar a la de $T$. trichiura. Resultados similares han sido señalados previamente $(11,27,31,35,36)$.

Algunos autores señalan que la prevalencia de T. trichiura y en general de los otros geohelmintos ha disminuido en las últimas décadas en las regiones urbanas debido a las mejoras en el saneamiento ambiental y en las condiciones de vida (42). Sin embargo, esto es parcialmente cierto ya que estudios recientes siguen demostrando elevadas tasas de prevalencia (31).

La mayoría de los niños parasitados estaba poliparasitado $(83,6 \%)$ lo que es frecuente que ocurra en áreas rurales y en general donde existan las condiciones que favorecen estas infecciones (30, $35,36)$. Además, es importante considerar que muchos de estos parásitos comparten la misma epidemiología y por lo tanto, en su transmisión juega un papel importante las condiciones ecológicas y de medio ambiente $(11,30)$.

Dentro de los protozoarios, B. hominis fue el más prevalente $(76,2 \%)$. Este controversial parásito intestinal, además de ser actualmente considerado un patógeno emergente, presenta elevadas tasas de prevalencia en diversos grupos de poblaciones (28, 43, 44). En escolares, su prevalencia es variable pero en general elevada. La aquí señalada es una de las más elevadas encontradas en escolares venezolanos $(28,30)$. En el estado Bolívar la prevalencia de $B$. hominis en escolares es de $16 \%$ aproximadamente (44).

Se debe resaltar la elevada prevalencia de algunos protozoarios no patógenos como E. coli (26,4\%) y Endolimax nana $(24,7 \%)$, que aunque tienen poca importancia desde el punto de vista clínico, son de gran relevancia epidemiológica ya que su presencia en las muestras de los escolares examinados es indicativo de contaminación fecal del agua y/o los alimentos (27).

También destacó el hallazgo de 12 casos de E. histolytica/E. dispar el cual es un protozoario infrecuente en el estado Bolívar $(32,45)$.

Aunque las parasitosis intestinales tienen en común la diseminación mediante la contaminación fecal, los modos de transmisión como elementos que componen la cadena epidemiológica han sido poco estudiados. Entre ellos, las manos y los respectivos depósitos subungueales de personas que no guardan las debidas nociones de higiene, 


\section{F Al Rumhein, J Sánchez, I Requena, Y Blanco, R Devera.}

representan la mayor fuente de vehiculación y diseminación de enteroparásitos (46).

Fueron analizadas 307 muestras de material subungueal de los mismos escolares sometidos al estudio coproparasitológico. Resalta el hecho de haber evaluado un gran número de muestras obteniendo un bajo índice de positividad $(3,6 \%)$, lo cual difiere a lo encontrado por otros autores $(14,15,19,46,47)$. Se ha sugerido que la presencia de formas parasitarias en el lecho subungueal no sólo depende de la edad sino principalmente de los hábitos higiénicos de la persona (20). Es por ello que a pesar de que los niños están poliparasitados y con cargas elevadas sí mantienen adecuadas medidas de higiene (lavado de manos, uñas cortas, etc.) los resultados serán negativos. A pesar de estas consideraciones tal vez ésta no sea la explicación del porqué se encontró tan poco número de casos de formas parasitarias en el depósito subungueal de estos niños.

Brasil ha sido pionero en investigaciones tendientes a demostrar la importancia del material subungueal como posible diseminador de enteroparásitos $(14,15,19,21,46,48)$. Campos (15) evaluó 130 habitantes de un Barrio de Natal, encontrando 16 casos con formas parasitarias en el depósito subungueal. Mello y col. (19) estudiaron 148 niños escolares de São Paulo, determinando una prevalencia de $16,2 \%$. Lima y col. (46), señalaron $14,3 \%$ de prevalencia evaluando 77 escolares, de una escuela de Santa Catarina al sur de Brasil.

En el presente estudio, se identificaron cinco especies de enteroparásitos en el material subungueal en los 11 casos positivos. A. lumbricoides fue el más prevalente, coincidiendo con los trabajos de Campos (14), Mello y col. (19), Lima y col. (46) y Oliveira y col. (47). El segundo parásito en frecuencia fue $E$. coli que también ha sido encontrado previamente $(14,48)$. Se identificaron dos casos con quistes de E. nana. Esta especie también ha sido encontrada en el lecho subungueal (20), pero en adultos manipuladores de alimentos. Los otros helmintos y protozoarios diagnosticados, como en otros estudios presentaron una baja prevalencia $(14,15,19,46-48)$. Cabe destacar la ausencia de huevos de E. vermicularis, que ha sido uno de los enteroparásitos más comúnmente encontrado en material subungueal $(14,15,19,21,23)$.

A pesar de diagnosticarse cinco especies distintas de enteroparásitos, la cantidad de formas parasitarias fue pequeña en todos los casos y, generalmente los quistes y/o huevos estaban parcialmente deformados, sin embargo, mantenían suficientes características que permitieron su identificación.

Al relacionar los parásitos presentes en el lecho subungueal con aquellos diagnosticados en las heces, no se encontró asociación estadísticamente significativa. En otros estudios a pesar de siempre encontrarse menor número de casos en las uñas en general los parásitos más prevalentes en heces también lo fueron en el depósito subungueal (48).

Por otro lado, Rincón de Heredia (20) sí encontró una asociación estadísticamente significativa, y concluyó que la fuente de contaminación principal de las uñas era la materia fecal de la misma persona. Resultado que contrasta con los hallazgos de la presente investigación.

De los 11 casos, en tres se diagnosticaron más de una especie parasitaria. La presencia de poliparasitismo en el lecho subungueal ha sido señalado pocas veces (19).

La determinación de 11 casos con formas parasitarias y 5 diferentes especies de helmintos y protozoarios en el lecho subungueal de escolares demuestra la importancia de este sitio como posible fuente de infección. Sin embargo, los resultados obtenidos en el grupo de niños estudiados, sugieren que el depósito subungueal no parece tener un papel principal como diseminador de enteroparásitos, debido al poco número de casos y a la baja carga parasitaria encontrada.

La alta prevalencia de enteroparasitosis encontrada en el presente estudio podría explicarse por las condiciones de insalubridad e inadecuado saneamiento ambiental, aunado al hacinamiento y a

\section{Revista Biomédica}




\section{Parasitosis intestinales en escolares.}

la falta de educación sanitaria existente entre los habitantes, lo cual conlleva al desarrollo de hábitos higiénicos inadecuados y una calidad de vida deficiente, facilitando de esta manera la diseminación de parásitos intestinales.

Todos los niños parasitados recibieron de forma gratuita tratamiento antiparasitario específico, gracias a la colaboración del Programa de Parasitosis Intestinal del estado Bolívar y el Colegio de Médicos del mismo estado.

Todavía hoy en el estado Bolívar, como en otras regiones de Venezuela, los parásitos intestinales en especial los geohelmintos, son un problema de salud pública en la población infantil. Es por ello, que la lucha contra las parasitosis no se ganará sólo tratando a los hospederos, sino con la educación sanitaria adecuada y mejoras socioeconómicas dirigidas a la etiología social de estas enfermedades $(31,35,37)$.

Desde el punto de vista preventivo, las campañas educativas a nivel de las escuelas ofrecen mayores y mejores resultados, siendo menos onerosas que otras medidas como las campañas de desparasitación masiva (6). Ésta debe ser una estrategia a ser aplicada en profundidad en todas las escuelas del estado Bolívar, y especialmente en la U.E.E. Teresa de la Parra, ante la imposibilidad de mejorar a corto plazo el marco socio-económico en el cual ocurren las parasitosis intestinales.

En conclusión, se determinó una elevada prevalencia de parásitos intestinales en las heces de los escolares, mientras que la prevalencia de formas parasitarias en el depósito subungueal fue bajo.

\section{AGRADECIMIIENTOS.}

A las licenciadas Hecmil González, Yolimar Jiménez, Natacha Rodríguez, Yubrimi Berhens Yamilet Lista y al Sr. Americo Berhens por su colaboración en las actividades de campo. A las directoras, subdirectoras, personal docente, empleados y obreros de la U.E.E. Teresa de La Parra por su cooperación en la elaboración de este trabajo. Al profesor Duglas Sánchez por la revisión del summary.

\section{REFERENCIAS.}

1.- OMS (Organización Mundial de la Salud). Infecciones intestinales por protozoos y helmintos. Edit. Gráficas Reunidas; 1981. Serie informes técnicos 666. pp 155.

2.- WHO (World Health Organization). WHO Expert Committee. Public health significance of intestinal parasitic infections. Bull WHO 1987; 65:575-88.

3.- Kvalsvig JD, Coopan RM, Connolly K The effects of parasite infections on cognitive processes in children. Ann Trop Med Parasitol 1991; 85:551-68.

4.- Savioli L, Bundy DAP, Tomkins A. Intestinal parasitic infections: a soluble public health problem. Trans R Soc Trop Med Hyg 1992; 86:353-4.

5.- Nokes C, Bundy DAP. Does helminth infection affect mental processing and educational achievement? Parasitol Today 1994; 10:14-8.

6.- Chan MS The global burden of intestinal nematode infections-fifty years on. Parasitol Today 1997; 13:43843.

7.- Sakti H, Nokes C, Hertanto WS, Hendratno S, Hall A, Bundy DAP, et al. Evidence for an association between hookworm infection and cognitive function in Indonesian school children. Trop Med Inter Health 1999; 4:322-34.

8.- Rey L. 1991. Parasitologia. 2da. ed. Rio de Janeiro: Edit. Guanabara-Koogan; 1991.p. 731.

9.- Jonnalagadda PR, Bhat RV. Parasitic Contamination of stored water used for drinking cooking in hyderabad. Southeast Asian J Trop Med Public Health 1995; 26:78994.

10.- Smith HV, Ahmad R. Protozoan parasites in the water. Parasitol Today 1997; 13:3-4.

11.- Morales GA, Pino L, Arteaga C, Matinella L, Rojas H. Prevalencias de las geohelmintiasis intestinales en 100 municipios de Venezuela (1898-1992). Rev Soc Bras Med Trop 1999; 32:263-70.

12.- Chieffi PP, Moretti IG, Foizer ACM, Nakagawa E, Gomes AC. Contribuição ao estudo da historia natural de enteroparasitoses em uma comunidade fechada. Rev Soc Bras Med Trop 1974; 8:87-91. 


\section{F Al Rumhein, J Sánchez, I Requena, Y Blanco, R Devera.}

13.- Silva Coelho LMP, Sobrino TA, Oliveira SM, Ikegami MT, Yoshizumi AM, et al. Ovos e larvas de helmintos nos sanitários de pré-escolas municipales de Sorocaba, SP e suas frequencias nas fezes de crianças. Rev Soc Bras Med Trop 1999; 32:647-52.

14.- Goulart EG, da Silva WR, Faraco BFC, de Moraes DS. Pesquisa de cistos e ovos de enteroparasitos do homen no deposito subungueal. Rev Bras Med 1966; 23:465-66.

15.- Campos CAM. O deposito subungueal como disseminador de enteroparasitos. Rev Soc Bras Med Trop 1974; 8:93-7.

16.- Carvalho DO. Frequência do Enterobius vermicularis nas crianças do Distrito Federal. Ciên Méd 1928; 6:117-8.

17.- Goulart EG. Diagnostico-tratamento da enterobiose. Rev Bras Med 1959; 16:756-60.

18.- Andrews J. The retention of Entamoeba histolytica cysts under finger nails. Am J Trop Méd 1934; 14: 439-41.

19.- Mello EB, Souza Jr, FL, Pádua HB, Campos MS, Tanabe TH. Encontro de ovos de helmintos e de cistos de protozoarios intestinais na região subungueal de crianças em idade escolar dos Municipios de Diadema e de Bragança Paulista, São Paulo. Rev Patol Trop 1978b; 7:4750.

20.- Rincón de Heredia W. Prevalencia de enteroparásitos en depósito subungueal y muestras fecal de manipuladores de alimentos en el "Hospital General del Sur de Maracaibo". Kasmera 1983; 11:65-77.

21.- Paço JM, Santos MA, Alves EL. Pesquisa de parasitos no conteuúdo subungueal em escolares de Goiânia. Rev Patol Trop 1991; 20:157-63.

22.- Guilherme AL, de Araujo, SM, Falavigna DL, Pupulim AR, Dias ML, de Oliveira HS., et al. Prevalencia de enteroparásitas em horticultores e hortaliças da Feira do Produtor de Maringá, Paraná. Rev Soc Bras Med Trop 1999; 32:405-11.

23.- Silva EMA, Nunes JFL, Wanderley AAS, Andrade GV, Abrantes PGL, Ursulino RST, et al. Pesquisa de ovos de Enterobius vermicularis na região subungueal em escolares da Cidade de Natal-RN. Rev Bras Anal Clin 2000; 32:176.
24.- Melvin DM, Brooke MM. Métodos de laboratorio para diagnóstico de parasitosis intestinales. $1^{\mathrm{a}} \mathrm{ed}$. México: Nueva Editorial Interamericana, S.A. 1971. p. 198.

25.- Morales G, Pino LA. Parasitología cuantitativa. $1^{\text {a }} \mathrm{ed}$. Caracas: Fondo Editorial Acta Cientifica Venezolana; 1987. p. 132.

26.- Chourio Lozano G, Heredia RW, Castellano M, Luzardo T, Meleán C. Prevalencia parasitaria en una comunidad suburbana del Distrito Maracaibo, estado Zulia. Kasmera 1988; 16:30-49.

27.- Díaz I, Duran TF. Prevalencia de parasitosis intestinales en alumnos de educación básica del Municipio Cacique Mara, Maracaibo-Estado Zulia. Kasmera 1990; 18:46-71.

28.- Beauchamp S, Flores T, Tarazón S. Blastocystis hominis: prevalencia en alumnos de una escuela básica. Maracaibo, Edo. Zulia. Venezuela. Kasmera 1995; 23:4367.

29.- Rivero Rodríguez Z, Chango Gómez Y, Iriarte Nava H. Enteroparásitos en alumnos de la Escuela Básica Dr. "Jesus María Portillo", Municipio Maracaibo, Edo. Zulia, Venezuela. Kasmera 1997; 25:121-44.

30.- Rivero Rodríguez Z, Chourio-Lozano G, Díaz I, Cheng R, Rucson G. Enteroparásitos en escolares de una institución pública del municipio Maracaibo, Venezuela. Invest Clin 2000; 41:37-57.

31.- Devera R, Niebla PG, Nastassi CJ, Velásquez AV, González MR. Prevalencia de Trichuris trichiura y otros enteroparásitos en siete escuelas del área urbana de Ciudad Bolívar, Estado Bolívar, Venezuela. Saber 2000; 12:41-7.

32.- Piña E, Muñoz J, Requena CI, León M, Devera R, Velásquez $\mathrm{V}$, et al. Prevalencia de parasitosis intestinales en escolares de la Unidad Educativa "Juan Vicente Cardoso", San Félix. Estado Bolívar, Venezuela. Memorias XVI Jornadas Científicas, Tecnológicas y Educativas de Guayana. 2-4 de noviembre de 2000. Ciudad Bolívar, Venezuela. 2000. p. 78-79.

33.- Calchi La Corte M, Chourio GL, Díaz I. Helmintiasis intestinales en niños de una comunidad marginal del Municipio Maracaibo, estado Zulia-Venezuela. Kasmera 1996; 24: 17-38.

\section{Revista Biomédica}




\section{Parasitosis intestinales en escolares.}

34.- Araujo M, Diaz AI, Chourio LG, Calchi M, Rivero RZ, Corzo G. Ascariasis. Correlación entre cargas parasitarias, estado nutricional y manifestaciones clínicas. Maracaibo, Edo. Zulia. Venezuela. Kasmera 1998; 26:61-90.

35.- Simoes M, Rivero Z, Díaz I, Carreño G, Lugo M, Maldonado A, et al. Prevalencia de enteroparásitos en una Escuela urbana en el Municipio San Francisco, estado Zulia, Venezuela. Kasmera 2000; 28:27-43.

36.- Rivero Rodríguez Z, Díaz I, Acurero E, Camacho MC, Medina M, Rios L. Prevalencia de parásitos intestinales en escolares de 5 a 10 años de un instituto del municipio Maracaibo, Edo. Zulia-Venezuela. Kasmera 2001; 29:15370.

37.- Botero D. Persistencia de Parasitosis intestinales endémicas en América Latina. Bull Of Sanit Panam 1981; 90:39-47.

38.- Ramos L, Salazar-Lugo R. Infestación parasitaria en niños de Cariaco-Estado Sucre, Venezuela y su relación con las condiciones socio-económicas. Kasmera 1997; 25:175-89.

39.- Chacín-Bonilla J, Bonilla E, Parra AM, Estevez J, Morales LM, Suarez H. Prevalence of Entamoeba histolytica and other intestinal parasites in a community from Maracaibo, Venezuela. Ann Trop Med Parasitol 1992; 86:373-80.

40.- Díaz I, Chourio G, Alvarez M, Añez O, Morón A, Romero E. Prevalencia de parásitos intestinales en el Barrio Teotiste de Gallegos de la Ciudad de Maracaibo, estado Zulia, Venezuela. Kasmera 1992; 20:73-93.

41.- Guevara R. Ornidazol en Giardiasis. Cuad Geog Med Guay 1986; 1:43-52.

42.- Atías, A. 1992. Tricocefalosis. En: Atías-Neghme, editores. Parasitología Clínica. 3ra ed. Chile: Publicaciones Técnicas Mediterráneo; 1992. p. 171-5.

43.- Torres P, Miranda JC, Flores L, Riquelme JM, Franjola $\mathrm{R}$, Pérez $\mathrm{J}$, et al. Blastocistosis y otras infecciones por protozoarios intestinales en comunidades humanas ribereñas de la cuenca del río Valdivia, Chile. Rev Inst Med Trop São Paulo 1992; 34:557-64.

44.- Devera R, Nastasi J, Niebla G, González R, Velásquez V. Prevalencia de infección por Blastocystis hominis en escolares de Ciudad Bolívar, Venezuela. Bol Chil Parasitol 1997; 52:77-81.
45.- Devera R. Ausencia de Entamoeba histolyticalE. dispar en Ciudad Bolívar, Estado Bolívar, Venezuela. Rev Biomed; 1998; 9:145-50.

46.- Lima LM, Visalli A, Reinert B. Pesquisa de enteroparasitas no conteudo subungueal de crianas matriculadas na escola Alfredo Domingos, no municipio del Balneãrio Camboriu, SC. Memorias del XV Congreso de Parasitologia. Bahia, Brasil; 1997. p. 130.

47.- Oliveira GC, Pereira CRC, Luz L. Parasitoses em crianas de creches da periferia de Goiania. Rev Soc Bras Med Trop 2000; 33 (Supl. I):146-7.

48.- Cancio CR, Dacai AR, Wanderley FS, Soares VL, Calheiros CM. Ocorrência de helmintoses e protozoozes em amostras fecais e de residuos do leito subungueal de crianças em favelas do municipio de Marechal Deodoro, Alagaos. Brasil. Memorias del XV Congresso de Parasitologia. Poços de Caldas, Brasil. 1999. p. 183. 